\title{
Monoclonal Gammopathy, Arthralgias, and Recurrent Fever Syndrome: A New Autoinflammatory Syndrome?
}

\author{
Alexandre Terré ${ }^{(i)}$, Alexis Talbot ${ }^{(i)}$, Camille Louvrier ${ }^{(\mathbb{D})}$, Jean Baptiste Picque,

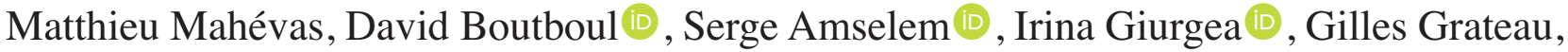 \\ and Sophie Georgin-Lavialle $\mathbb{B}^{\mathbb{B}}$, for the French Network of Dysimmune Disorders \\ Associated with Hemopathies
}

\begin{abstract}
Objective. To describe a new autoinflammatory syndrome with recurrent fever and monoclonal gammopathy that differs from Schnitzler syndrome.

Methods. We conducted a retrospective study of patients with monoclonal gammopathy and recurrent fever of unknown origin.

Results. Five patients were studied; median age at onset of symptoms was 44 years. Median frequency of fever attacks was 6 episodes per year. In the absence of treatment, the median duration of fevers was 3 days.

Conclusion. This new autoinflammatory syndrome is defined by an association among monoclonal gammopathy, arthralgias, and recurrent fever. (First Release August 15 2019; J Rheumatol 2019;46:1535-9; doi:10.3899/jrheum.181204)
\end{abstract}

Key Indexing Terms:

INNATE IMMUNE RESPONSE SCHNITZLER SYNDROME PARAPROTEINEMIAS

Autoinflammatory diseases were recently defined by an international consortium of experts as "clinical disorders caused by defect(s) or dysregulation of the innate immune system, characterized by recurrent or continuous inflammation

From the Sorbonne Université, AP-HP, Hôpital Tenon, Service de Médecine Interne, Centre de Référence des Maladies Auto-inflammatoires et des Amyloses d'Origine Inflammatoire (CEREMAIA); Service d'Immuno-hélatologie, Hôpital St. Louis; INSERM UMRS-933, et Laboratoire de Génétique, Hôpital Trousseau, AP-HP, Faculté de Médecine, Sorbonne Université, Paris; Service de Médecine Interne, Centre Hospitalier d'Auxerre, Auxerre; Service de Médecine Interne, Centre Hospitalier Universitaire (CHU) Henri Mondor; and Service d'Immunologie Clinique, Hôpital St. Louis, Paris, France.

A. Terré, MD, Sorbonne Université, AP-HP, Hôpital Tenon, Service de Médecine Interne, CEREMAIA; A. Talbot, MD, Service d'Immunohélatologie, Hôpital St. Louis; C. Louvrier, PharmD, INSERM UMRS-933, et Laboratoire de Génétique, Hôpital Trousseau, AP-HP, Faculté de Médecine, Sorbonne Université; J.B. Picque, MD, Service de Médecine Interne, Centre Hospitalier d'Auxerre; M. Mahévas, MD, Service de Médecine Interne, CHU Henri Mondor; D. Boutboul, MD, Service d'Immunologie Clinique, Hôpital St. Louis; S. Amselem, MD, PhD, INSERM UMRS-933, et Laboratoire de Génétique, Hôpital Trousseau, AP-HP, Faculté de Médecine, Sorbonne Université; I. Giurgea, MD, PhD, INSERM UMRS-933, et Laboratoire de Génétique, Hôpital Trousseau, AP-HP, Faculté de Médecine, Sorbonne Université; G. Grateau, MD, PhD, Sorbonne Université, AP-HP, Hôpital Tenon, Service de Médecine Interne, CEREMAIA, and INSERM UMRS-933, et Laboratoire de Génétique, Hôpital Trousseau, AP-HP, Faculté de Médecine, Sorbonne Université; S. Georgin-Lavialle, $M D, P h D$, Sorbonne Université, AP-HP, Hôpital Tenon, Service de Médecine Interne, CEREMAIA, and INSERM UMRS-933, et Laboratoire de Génétique, Hôpital Trousseau, AP-HP, Faculté de Médecine, Sorbonne Université.

Address correspondence to Dr. S. Georgin-Lavialle, Internal Medicine Unit, Tenon Hospital, 20 rue de la Chine, 75020 Paris, France.

E-mail:sophie.georgin-lavialle@aphp.fr

Accepted for publication February 15, 2019. (elevated acute-phase reactants; APR) and the lack of a primary pathogenic role for the adaptive immune system"1. The main symptoms of autoinflammatory diseases are fever, cutaneous rash, arthritis or arthralgia, and abdominal pain.

Schnitzler syndrome is an autoinflammatory disease with recurrent fever, secondary to dysregulation of the interleukin 1 (IL-1) pathway ${ }^{2,3,4}$. The cornerstones of Schnitzler syndrome are a chronic urticarial rash and a monoclonal IgM or less frequently, IgG gammopathy ${ }^{3}$. It remains unclear whether the monoclonal gammopathy causes IL-1 pathway dysregulation and leads to symptoms or whether chronic dysregulation of the IL-1 pathway leads to development of a monoclonal gammopathy.

Despite this lack of understanding, the association between an autoinflammatory syndrome and monoclonal gammopathy is very interesting and could lead to the characterization of a new group of autoinflammatory diseases. Our aim was to describe a new autoinflammatory syndrome in which recurrent fever is associated with a monoclonal gammopathy and does not meet the criteria for Schnitzler syndrome ${ }^{3}$.

\section{MATERIALS AND METHODS}

We performed a retrospective multicenter study coordinated by the French reference center for autoinflammatory diseases (CEREMAIA). Patients from the CEREMAIA database with suspected autoinflammatory diseases who presented with recurrent fever and a monoclonal gammopathy were selected and their files were reviewed. To describe the new autoinflammatory syndrome, we applied the following inclusion criteria: (1) recurrent fever of unknown origin with at least 3 episodes of fever in more than 6 months and occurring with an elevated C-reactive protein (CRP); (2) the presence of 1 of the following symptoms during fever episodes: bone pain, arthralgia or

Personal non-commercial use only. The Journal of Rheumatology Copyright $\subset$ (2019. All rights reserved. 
arthritis, myalgia, abdominal pain, cutaneous rash; and (3) the presence of a monoclonal gammopathy by blood protein electrophoresis without evidence for multiple myeloma. To confirm the unknown origin of the fever, patients must have had a clinical examination including the following: blood cultures, human immunodeficiency virus, hepatitis $\mathrm{C}$ virus and hepatitis $\mathrm{B}$ virus serology, thoracoabdominal-pelvic computed tomography for neoplasia, and antinuclear and antineutrophil cytoplasmic antibody testing to exclude autoimmune disease. Genetic testing for autoinflammatory diseases was not required.

Patients with a definite autoimmune or autoinflammatory disease, especially Schnitzler syndrome, as well as an infectious or a neoplastic disease, were not included in this study.

All patients provided informed consent, and the study followed the requirements of the Helsinki Declaration. Ethical board approval was not required in accord with French law and our institutional review board.

\section{RESULTS}

The CEREMAIA database included 751 patients with an autoinflammatory disease. Sixteen patients displayed monoclonal gammopathy and recurrent fever but no defined diagnosis. Eleven were excluded from the final description because of genetically confirmed monogenic recurrent fever $(\mathrm{n}=2)$, hypocomplementemic urticarial vasculitis $(\mathrm{n}=1)$, fulfillment of the criteria for Schnitzler syndrome $(n=6)$, recent diagnosis of kidney neoplasm $(\mathrm{n}=1)$, or loss to followup $(\mathrm{n}=1$; Figure 1$)$.

Syndrome description. The 5 remaining patients met all the inclusion criteria; their features are summarized in Table 1. Three patients $(60 \%)$ were men. The median age at the beginning of symptoms was 44 years (range 30-71 yrs). The median frequency was 6 episodes of fever per year (range 3-12 episodes per yr). The median duration of the fevers without treatment was 3 days (extreme values 2 to 12). The most frequently associated symptom was muscle pain, which was found in all patients $(100 \%)$. Arthralgia was the second most frequent symptom, found in 4 patients $(80 \%)$, and involved the spine (40\%) or multiple peripheral joints (40\%). The phenotypes seemed to be stable during followup, and no spontaneous remission was mentioned. No patient had bone pain, abdominal pain, arthritis, or skin involvement. There was no indication of bone remodeling on plain radiographs. One patient had recurrent episodes of exudative neutrophilic aseptic pleuritis during fever attacks, with no evidence for malignancy in a pleural biopsy. The most frequent isotype for the monoclonal gammopathy was $\mathrm{IgG}$, which was found in 3 patients $(60 \%)$, and the amount ranged from "barely detectable" to $19.8 \mathrm{~g} / \mathrm{l}$ (median value $6.6 \mathrm{~g} / \mathrm{l}$ ). For the light chain, $\lambda$ was the most frequent and was found in 3 patients $(60 \%)$. There was no type other than IgG or IgM. All patients had elevated polymorphonuclear values and CRP with a median of $156 \mathrm{mg} / \mathrm{l}$ (range $84-250 \mathrm{mg} / \mathrm{l}$ ) during fever attacks.

Followed at the center for an autoinflammatory disease: 751 patients

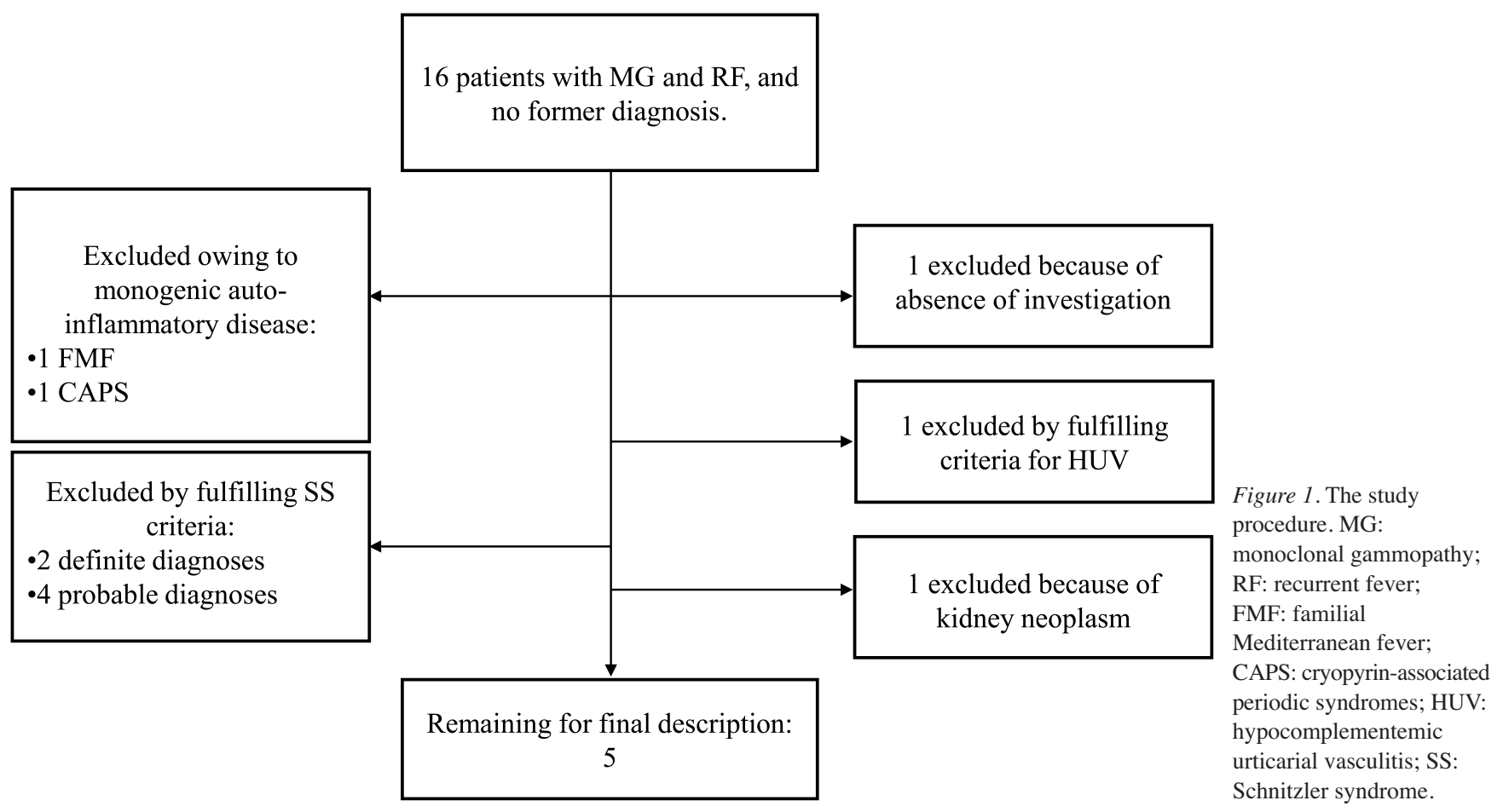

Personal non-commercial use only. The Journal of Rheumatology Copyright @ 2019 . All rights reserved. 
Table 1. Main clinical and biological features of patients.

\begin{tabular}{|c|c|c|c|c|c|c|c|c|c|}
\hline Patient & Sex & Ethnicity & $\begin{array}{l}\text { Family } \\
\text { History }\end{array}$ & $\begin{array}{l}\text { Age at Onset } \\
\text { of Symptoms, yrs }\end{array}$ & $\begin{array}{c}\text { Frequency of } \\
\text { Fever Attacks, } \\
\text { per yr }\end{array}$ & $\begin{array}{l}\text { Duration of } \\
\text { Fever, days }\end{array}$ & $\begin{array}{l}\text { Joint Pain } \\
\text { Location }\end{array}$ & $\begin{array}{l}\text { Muscle } \\
\text { Pain }\end{array}$ & $\begin{array}{c}\text { Type of } \\
\text { Monoclonal } \\
\text { Gammopathy }\end{array}$ \\
\hline 2 & $\mathrm{~F}$ & White & Mother, psoriasis & 30 & 12 & 4 & Spine & Yes & $\operatorname{IgG} \lambda$ \\
\hline 3 & $\mathrm{~F}$ & White & None & 71 & 3 & 2 & None & Yes & $\operatorname{IgG} \kappa$ and $\operatorname{IgM} \kappa$ \\
\hline 4 & M & White & None & 39 & 12 & 3 & Polyarthralgia & Yes & $\operatorname{IgG} \lambda$ \\
\hline
\end{tabular}

SLE: systemic lupus erythematosus.

Molecular investigations of monogenic autoinflammatory diseases were performed for 3 of them: the $M E F V$ (exon 10) and TNFRSF 1A (exons 2, 3, and 4) genes were analyzed by Sanger sequencing for 1 patient, and for the 2 others, the TNFRSF1A, MEFV, NLRP3, MVK, and NLRC4 genes were analyzed using next-generation sequencing. No deleterious germ line or somatic mutations were identified in these 3 patients.

Treatments. Two patients were treated with $1 \mathrm{mg}$ colchicine per day, without success. One patient had been treated with $0.7 \mathrm{mg} / \mathrm{kg} /$ day prednisolone during fever attacks, without success. Two patients had been treated with the IL-1 receptor antagonist anakinra during attacks and had a good response.

\section{DISCUSSION}

We describe 5 patients with a new clinical and biological autoinflammatory syndrome. This syndrome should be considered only after careful examination of differential diagnosis, especially concerning Schnitzler syndrome. This syndrome occurs in adults older than age 30 years who have no prior symptoms. Symptoms included recurrent fevers lasting 3 to 12 days, arthralgia involving the peripheral joints or the spine, and myalgia. This condition could also be associated with pleuritis. Biologically, the syndrome is defined by a monoclonal gammopathy of IgG or IgM without specificity for the light chain, as well as elevated CRP during fever attacks. These features are summarized in Figure 2A.

This syndrome shares common features with Schnitzler syndrome, including recurrent fever, arthralgia, and monoclonal gammopathy. The main difference in our cases is the absence of skin involvement, which is a major criterion for Schnitzler syndrome. Without chronic urticaria, a diagnosis of Schnitzler syndrome cannot be confirmed ${ }^{3}$. Interestingly, there was no sign of osteosclerosis or bone remodeling in our cases, whereas it is an important finding in Schnitzler syndrome.

Schnitzler syndrome is believed to be an autoinflammatory syndrome because of recurrent fever, elevated APR, dysregulation of proinflammatory cytokines, and the efficiency of anti-IL-1 biotherapies ${ }^{2,4,5,6}$. The new syndrome described here is characterized by recurrent fever, elevated APR, and the efficacy of anti-IL-1 therapy. Systematic genetic testing or a cytokine profile study has not been done for this new syndrome, but there is no evidence for autoimmunity or infectious disease, so an autoinflammatory disease seems likely. The significance of the monoclonal gammopathy remains unclear. Hypotheses can be formed from what is known about Schnitzler syndrome. The first hypothesis is that the monoclonal isotype has autoantibody activity that triggers an IL-1 cascade. This hypothesis is supported in Schnitzler syndrome by the presence of anti-IL- $1 \alpha$ antibodies in some patients, which may increase the half-life of this proinflammatory cytokine ${ }^{7}$, as well as the efficacy of treatments that reduce the amount of monoclonal gammopathy ${ }^{8}$. However, this hypothesis is currently not supported, and the presence and pathogenic expression of anti-IL-1 $\alpha$ antibodies have not been confirmed. The second hypothesis, which is popular in Schnitzler syndrome research, is that symptoms are secondary to an inflammatory cytokine dysregulation that results in development of a monoclonal gammopathy. This hypothesis is supported by delayed development of monoclonal gammopathy in some cases ${ }^{9}$, and by the discovery of somatic mosaic mutations of the NLRP3 gene restricted to a myeloid lineage in 2 patients affected with variant-type Schnitzler syndrome ${ }^{10}$; however, this finding was not confirmed in a recent larger series ${ }^{11}$. Next-generation sequencing was performed in 2 patients, and no deleterious mutations in the MEFV, MVK, NLRP3, NLRC4, or TNFRSF 1A genes were identified. With no pathogenetic explanation for this syndrome, the disorder seems to fall on the border between monoclonal gammopathies and autoinflammatory disease (Figure 2B).

However, the association of a monoclonal gammopathy with autoinflammatory symptoms in Schnitzler syndrome and in the syndrome described here does not seem to be a coincidence, and a new category of syndrome should be created that could be termed monoclonal gammopathy of inflammatory significance based on other categories, as has been done for monoclonal gammopathy of renal significance and monoclonal gammopathy of cutaneous significance ${ }^{12}$. This group would include Schnitzler syndrome and the syndrome described in this report.

Another secondary but interesting point is that Schnitzler syndrome remains underdiagnosed. Among our 16 patients

Personal non-commercial use only. The Journal of Rheumatology Copyright @ 2019 . All rights reserved. 

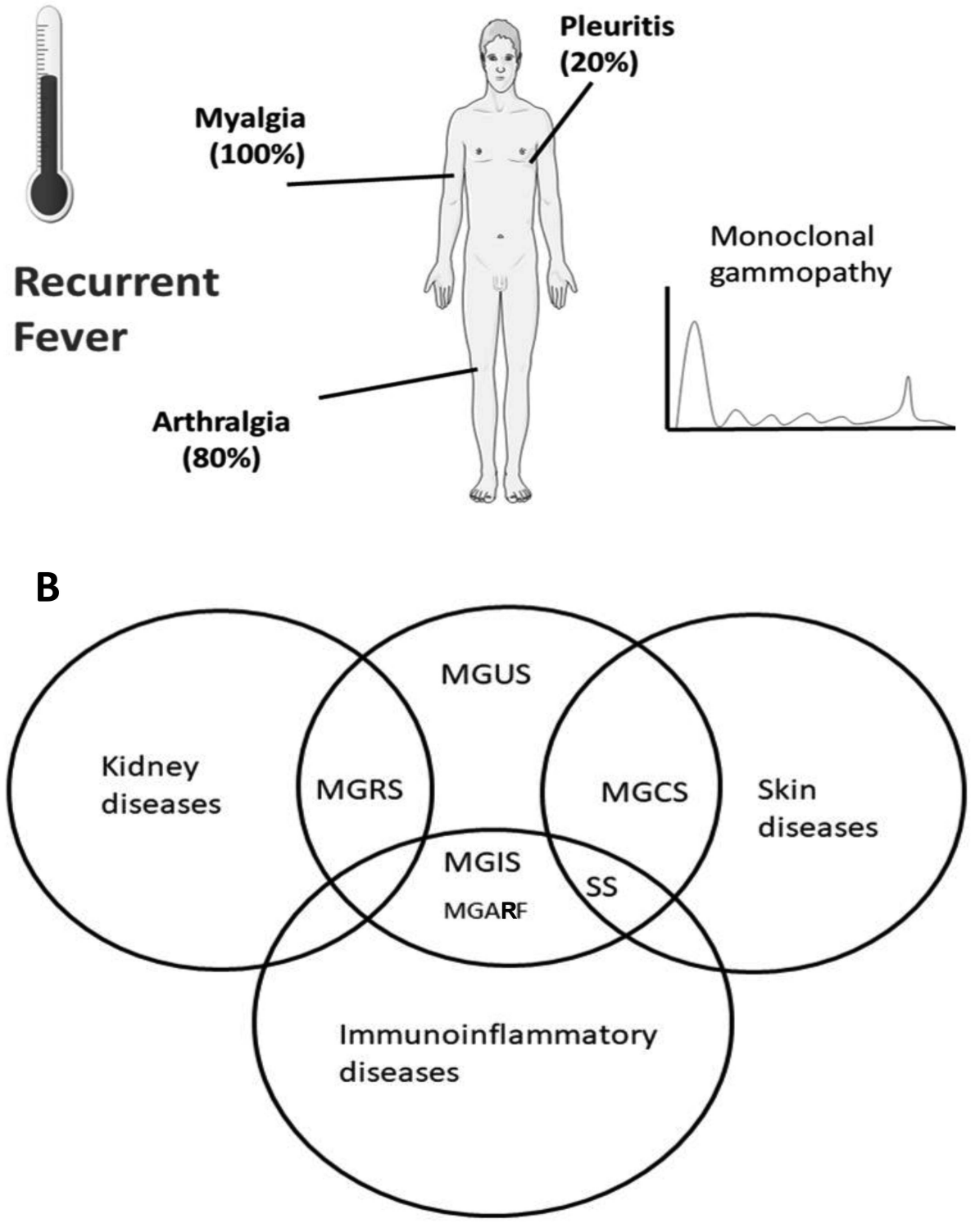

Figure 2. A. Main clinical features of the patients. B. Proposal for a nosological classification of this entity in the spectrum of autoinflammatory diseases and monoclonal gammopathies. MGUS: monoclonal gammopathy of undetermined significance; MGRS: monoclonal gammopathy of renal significance; MGCS: monoclonal gammopathy of cutaneous significance; MGIS: monoclonal gammopathy of inflammatory significance; MGARF: monoclonal gammopathy, arthralgias, and recurrent fever syndrome; SS: Schnitzler syndrome.

with recurrent fever and a monoclonal gammopathy, 6 $(37.5 \%)$ fulfilled the diagnostic criteria for Schnitzler syndrome; all had monoclonal gammopathy with an $\mathrm{IgG}$ isotype. Despite inclusion of an IgG monoclonal gammopathy in the diagnostic criteria ${ }^{3}$, this variant of Schnitzler syndrome is not always recognized by clinicians.

We describe the first series of a new gammopathy-related autoinflammatory syndrome that is different from Schnitzler syndrome owing to the absence of skin involvement. This new syndrome could be termed monoclonal gammopathy, arthralgias, and recurrent fever syndrome, or MGARF, which includes the 3 main features of the syndrome.

\section{REFERENCES}

1. Ben-Chetrit E, Gattorno M, Gul A, Kastner DL, Lachmann HJ Touitou I, et al. Consensus proposal for taxonomy and definition of the autoinflammatory diseases (AIDs): A Delphi study. Ann Rheum Dis 2018;77:1558-65.

2. Gusdorf L, Lipsker D. Schnitzler syndrome: a review. Curr Rheumatol Rep 2017;19:46.

3. Simon A, Asli B, Braun-Falco M, De Koning H, Fermand J-P, 
Grattan C, et al. Schnitzler's syndrome: diagnosis, treatment, and follow-up. Allergy 2013;68:562-8.

4. de Koning HD, Schalkwijk J, Stoffels M, Jongekrijg J, Jacobs JF, Verwiel E, et al. The role of interleukin-1 beta in the pathophysiology of Schnitzler's syndrome. Arthritis Res Ther 2015; 17:187.

5. Néel A, Henry B, Barbarot S, Masseau A, Perrin F, Bernier C, et al. Long-term effectiveness and safety of interleukin-1 receptor antagonist (anakinra) in Schnitzler's syndrome: A French multicenter study. Autoimmun Rev 2014;13:1035-41.

6. Krause K, Tsianakas A, Wagner N, Fischer J, Weller K, Metz M, et al. Efficacy and safety of canakinumab in Schnitzler syndrome: a multicenter randomized placebo-controlled study. J Allergy Clin Immunol 2017;139:1311-20.

7. Saurat JH, Schifferli J, Steiger G, Dayer JM, Didierjean L. Anti-interleukin-1 alpha autoantibodies in humans: characterization, isotype distribution, and receptor-binding inhibition - higher frequency in Schnitzler's syndrome (urticaria and macroglobulinemia). J Allergy Clin Immunol 1991;88:244-56.
8. Aouba A, Pressiat C, Pricopi M, Georgin-Lavialle S, Boue F, Lievre-Castilla M-A, et al. Complete remission of Schnitzler syndrome and Waldenström macroglobulinemia under rituximab-cyclophosphamide-dexamethasone. Dermatology 2015;230:18-22.

9. Mulla E, Neame R. Delayed development of the $\operatorname{IgM}$ paraprotein in Schnitzler's syndrome. Scand J Rheumatol 2015;44:521-2.

10. de Koning HD, van Gijn ME, Stoffels M, Jongekrijg J, Zeeuwen PL, Elferink MG, et al. Myeloid lineage-restricted somatic mosaicism of NLRP3 mutations in patients with variant Schnitzler syndrome. J Allergy Clin Immunol 2015;135:561-4.

11. Rowczenio DM, Pathak S, Arostegui JI, Mensa-Vilaro A, Omoyinmi $\mathrm{E}$, Brogan $\mathrm{P}$, et al. Molecular genetic investigation, clinical features, and response to treatment in 21 patients with Schnitzler syndrome. Blood 2018;131:974-81.

12. Lipsker D. Monoclonal gammopathy of cutaneous significance: review of a relevant concept. J Eur Acad Dermatol Venereol 2017;31:45-52. 